\title{
Concreto reforzado con textil para el refuerzo y rehabilitación de columnas de hormigón armado
}

\author{
Textile Reinforced Concrete TRC for strengthening and rehabilitation of \\ RC-Columns
}
Betão têxtil reforçado para reforço e reabilitação de pilares de betão armado

\author{
Alejandro Gustavo Colque Romero \\ a.colque@structengout.com \\ ORCID 0000-0002-7923-8083 \\ Universidad Privada Boliviana, La Paz-Bolivia
}

Artículo recibido 10 septiembre 2020 | Arbitrado del 8 al 19 octubre 2020 | Publicado 1 enero 2021

RESUMEN

\begin{abstract}
Los avances tecnológicos proponen nuevas tecnologías en la rehabilitación de columnas estructurales, algunas de ellas incorporan textil como material de refuerzo. En este sentido, el objetivo de esta investigación es aplicar concreto reforzado con textil para reforzar y rehabilitar columnas de hormigón armado, con materiales existentes en Bolivia. Se describen 6 escenarios bajo normas Eurocódigo y ACI, para evaluar la relación costoeficiencia de combinar concreto reforzado con 3 materiales diferentes: textil de fibra de vidrio, textil de fibra de carbono y manta de carbono. Como resultado se comprueba que la mejor combinación es "concreto de grano fino con fibra textil de vidrio o carbono", dado que incrementa la capacidad de carga y eleva la resistencia a fallas por pandeo, cortante, deformación y traslape. Asimismo, reduce el precio. Se concluyó que, en condiciones bolivianas, es posible reducir costos y elevar eficiencia usando concreto reforzado con textil para reforzar y rehabilitar columnas de hormigón armado.
\end{abstract}

Palabras clave: Hormigón armado; cálculo estructural; fibra de vidrio; fibra de carbono; resistencia de materiales
ABSTRACT

RESUMO
Technological advances propose new technologies in the rehabilitation of structural columns, some of them incorporate textile as a reinforcing material. The objective of this research is to apply textile reinforced concrete to reinforce and rehabilitate reinforced concrete columns, with existing materials in Bolivia 6 scenarios are described complying Euro-code and ACI standards, to evaluate the cost-efficiency relationship of combining reinforced concrete with 3 different materials: fiberglass textile, carbon fiber textile and carbon blanket. As a result, it is found that the best combination is "fine-grained concrete with glass or carbon textile fiber", since it increases the load capacity and increases the resistance to failures due to buckling, shear, deformation, and overlap. It also reduces the price. It is concluded that, considering Bolivian conditions, it is possible to reduce costs and increase efficiency by using concrete reinforced with textile to reinforce and rehabilitate reinforced concrete columns.

Key words: Reinforced concrete; structural calculation; fiberglass; Carbon fiber; strength of materials
Os avanços tecnológicos propõem novas tecnologias na reabilitação de pilares estruturais, algumas delas incorporando o têxtil como material de reforço. 0 objetivo desta pesquisa é aplicar concreto armado têxtil para reforçar e reabilitar pilares de concreto armado, com materiais existentes na Bolívia. 6 cenários são descritos nos padrões Euro-code e ACI, para avaliar a relação custo-eficácia da combinação de concreto armado com 3 materiais diferentes: fibra de vidro têxtil, fibra de carbono têxtil e manta de carbono. Como resultado, verificase que a melhor combinação é "concreto de granulação fina com fibra de vidro ou fibra de carbono", pois aumenta a capacidade de carga e aumenta a resistência a falhas por flambagem, cisalhamento, deformação e sobreposição. Também reduz o preço Conclui-se que, nas condições bolivianas, é possível reduzir custos e aumentar a eficiência com o uso de concreto armado com tecido para reforçar e reabilitar pilares de concreto armado.

Palavras-chave: Concreto reforçado; cálculo estrutural; fibra de vidro: fibra de carbono; resistência de materiais 


\section{INTRODUCCIÓN}

En ingeniería civil, el concreto reforzado con acero es el material compuesto más importante utilizado en aplicaciones estructurales. La combinación de una alta fuerza de compresión por parte del concreto y la alta fuerza de tensión por parte del acero de refuerzo permiten una apropiada capacidad de carga para muchas aplicaciones en la industria de la construcción. Cada vez se hace más frecuente que, al llegar al final de la vida útil teórica de las estructuras, estas muestran aún excelentes prestaciones $\mathrm{y}$, en consecuencia, debe considerarse la posibilidad de reutilizar la estructura introduciendo refuerzos o realizando reparaciones que restauren las condiciones de durabilidad. Desde el punto de vista resistente, si lo que se plantea es un cambio de uso que exija nuevas capacidades, es posible plantearse hoy día soluciones de refuerzo en pilares que, con costos mínimos en comparación con los de demolición y reconstrucción, resuelvan a la perfección el problema planteado.

La mejora de la estructura para su reutilización se asocia al refuerzo, ya que permite aprovechar las características mecánicas existentes para generar un nuevo sistema de mayor prestación. El refuerzo de estructuras existentes es una solución de reutilización sostenible, a igualdad de prestaciones en comparación con la obra nueva.

Ambiciosas investigaciones en búsqueda de optimización y modernización han desarrollado un nuevo material compuesto, el "Concreto Reforzado con Textil" conocido por sus siglas en inglés T.R.C. (Textile Reinforced Concrete) donde telas biaxiales y multiaxiales se utilizan en combinación con un "Concreto de Grano Fino" F.G.C. (Fine Grained Concrete), Hegger (2008). Esto permite el diseño de elementos estructurales muy delgados con altas prestaciones en compresión y tensión. El uso de textiles, principalmente hechos de Fibra de Vidrio Alcalino Resistente (vidrioAR) y otras veces de materiales como Carbón y Aramida, las cuales son colocadas en la dirección principal del esfuerzo del compuesto, permitiendo una gran efectividad en comparación con el uso de fibras cortas distribuidas aleatoriamente en el ya conocido Concreto Reforzado con Vidrio (GRC). Sin embargo, como el TRC es un material innovador, se están realizando investigaciones con información detallada para el diseño seguro de estructuras con soporte de carga, por lo tanto, pruebas ambiciosas en diferentes proyectos de investigación se llevan a cabo, Cubillos (2019).

Es importante resaltar estudios de dos universidades alemanas, la Universidad de Aachen presentó bases técnicas para el desarrollo de una nueva tecnología, y la Universidad de Dresden se enfocó en los refuerzos textiles para fortalecimiento $\mathrm{y}$ reparación de estructuras. Ambas investigaciones han establecido mecanismos básicos con respecto a la durabilidad, características de unión, así como la capacidad de carga dentro de los lineamientos y estándares para el dimensionamiento de tales elementos de estructura delgada. A la par de estos dos grandes proyectos de investigación, también se tienen trabajos de investigación en Israel, Estados Unidos, Grecia, Bélgica, Reino Unido y Canada, Schanack (2006). Asimismo, un estudio de la Universidad Católica Boliviana, aporta con pautas sobre 
la aplicación de polímeros reforzados con fibra de carbono para el refuerzo de hormigón, Butrón (2011).

El presente documento tiene como objetivo aplicar concreto reforzado con textil para reforzar y rehabilitar columnas de hormigón armado, en el contexto y condiciones de materiales existentes en Bolivia. Se estudió el comportamiento de las columnas reforzadas con TRC, a partir del estudio de la literatura técnica existente sobre el tema, asimismo, se hizo un cálculo experimental que permitió abordar esta tarea con elementos de encamisado de pequeño espesor, materializados con micro-hormigones (concreto de grano fino) de altas prestaciones y con criterios sencillos y prácticos tanto de armado como de tratamiento de la aludida interfaz.

La investigación se justifica porque el sistema de refuerzo propuesto es competitivo con relación a otros sistemas de refuerzo de columnas (polímeros reforzados con fibra de carbono epóxico), por tanto, significa ahorro en el rubro de la construcción. Además, el estudio ha sido valorado con materiales disponibles localmente, por tanto, es la primera solución práctica aplicable a la construcción en Bolivia.

Realizada la revisión teórica se expone que, de acuerdo con Brameshuber (2006), el confinamiento se aplica generalmente a los miembros de compresión, para mejorar su capacidad de carga o, en casos de modernización sísmica, para aumentar su ductilidad. Las técnicas tradicionales de confinamiento confían en los estribos de acero, encamisados de acero o CFRP (polímeros reforzados con fibra de carbono) para su reforzamiento. En problemas sísmicos, existen mejoras técnicas (ya sea de fortalecimiento o de refuerzo), basadas en el aumento de la presión de confinamiento.

Según Alzate (2012), existen varias técnicas para la aplicación de CFRP en elementos de hormigón, pueden clasificarse en tres grupos: wrapping o envoltura, bobinado atomístico y elementos prefabricados. Por otra parte, en los últimos años, la combinación de los textiles con concreto de alta resistencia ha llevado al desarrollo de la técnica llamada refuerzo con TRC, que es prometedora para el revestimiento de miembros de hormigón armado, así como para el fortalecimiento de mampostería no reforzada. Hasta el momento existen 3 métodos de refuerzo con TRC: laminación, pulverización e inyección.

El diseño de materiales compuestos de refuerzo para estructuras de hormigón armado debe seguir la filosofía de los códigos de diseño relevantes Euro-Códigos en Europa y ACI en Estados Unidos para la verificación del estado último y los estados límite de servicio. El Euro-código aplica factores de reducción de resistencia para cada material, modifica las cargas que actúan sobre las estructuras mediante la aplicación de factores de carga. Estos factores se encuentran en rangos entre 1,00 a 1,35 para acciones permanentes (cargas sostenidas) y entre 1,00 a 1,50 para acciones variables (cargas temporales). En el momento de la asignación de factores de combinaciones de carga, se debe considerar la probabilidad de aparición simultánea de nuevas cargas, EUROCODE (2004). Los códigos ACI aplican factores de reducción de resistencia de la misma forma que el Euro-código, aunque no asigna uno para cada material individual, utiliza en cambio 
factores de reducción de capacidad mayor (representados como $\varphi$ ). Consideran factores de aplicación de carga como parte de lo que está descrito como combinaciones de carga (o casos de carga). Los casos de carga toman en cuenta la probabilidad de dos o más tipos de carga actuando sobre la estructura al mismo tiempo, ACI (2005).

\section{MÉTODO}

El estudio tiene alcance descriptivo, investiga diferentes maneras de incorporar nuevas tecnologías enfocadas a la rehabilitación de columnas estructurales de hormigón armado. Se consideró las características de materiales reales existentes en Bolivia.

Se analiza las virtudes del concreto reforzado con 3 materiales diferentes: textil de fibra de vidrio, textil de fibra de carbono y manta de carbono. En cada uno de los casos se evalúa la relación costo-eficiencia, tomando como parámetros las características de: a) resistencia a la tracción última, b) módulo de elasticidad, c) deformación de rotura, d) número de capas de refuerzo, e) espesor de la capa final, f) incremento de carga en [kN], g) incremento de carga en [\%] $\mathrm{y}, \mathrm{h}$ ) precio por el refuerzo.

Estas características fueron evaluadas considerando su aplicación para reforzar columnas de hormigón armado con base rectangular y circular. Los resultados se obtuvieron al comparar sistemáticamente los siguientes 6 escenarios: 1) Refuerzo por confinamiento con TRC para columna rectangular con textil de fibra de vidrio-AR, según Euro-códigos. 2) Refuerzo por confinamiento con TRC para columna circular con fibra textil de vidrio-AR, según Euro-códigos. 3) Refuerzo por confinamiento con TRC para columna rectangular con textil de fibra de carbono, según Euro-códigos. 4) Refuerzo por confinamiento con TRC para columna circular con textil de fibra de carbono, según Euro-códigos. 5) Refuerzo con CFRP por confinamiento para una columna rectangular, según norma ACI. 6) Refuerzo con FRP por confinamiento para una columna circular, según norma ACI.

El cálculo estructural realizado aplica los principios, parámetros y valores establecidos por la norma vigente en Europa "Euro-código", Eurocode (2004)., según cuadro 1. Asimismo, realiza los mismos cálculos aplicando los parámetros establecidos por la normativa definida por ACI, ACI (2005), vigente en Estados Unidos, según cuadro 2 
Cuadro 1. Procedimiento y valores considerados (según Eurocode).

\begin{tabular}{ll}
\hline Procedimiento & \multicolumn{1}{c}{ Fórmula } \\
\hline $\begin{array}{l}\text { Cálculo del } \\
\begin{array}{l}\text { Espesor de TRC } \\
\text { para } \\
\text { Confinamiento }\end{array}\end{array}$ & $\begin{array}{l}f_{f d e}=n_{e} * f_{f d} \\
\mathrm{n}_{\mathrm{e}} \text { factor de reducción que para interiores es } 0.9\end{array}$ \\
$\begin{array}{l}\text { Cálculo del } \\
\text { módulo de } \\
\text { elasticidad del } \\
\text { hormigón }\end{array}$ & $\begin{array}{l}E_{c}=9,5 \cdot\left(f_{c k}+8\right)^{1 / 3} \\
\mathrm{f}_{\text {ek: }} \text { la resistencia característica del hormigón a los } 28 \text { días está dado por la } \\
\text { formula, }\end{array}$ \\
& $\begin{array}{l}\mathrm{f}_{\mathrm{ek}}=\mathrm{m}_{\mathrm{f}}\left(1-\mathrm{k}_{\mathrm{n}} * \mathrm{vf}_{\mathrm{f}}\right) .\end{array}$
\end{tabular}

Cálculo de la resistencia a la compresión del hormigón Cálculo del radio volumétrico en cada lado de la sección transversal de la columna

$$
f_{c d}=\frac{f_{c k}}{1,5}
$$

Para para columna rectangular con textil de fibra de vidrio-AR y para textil de fibra de carbono

$$
\rho_{f, b}=\frac{2 \cdot t_{f}}{d} \quad y \quad \rho_{f, d}=\frac{2 \cdot t_{f}}{b}
$$

Para el cálculo se toma el lado de mayor influencia

Para columna circular con fibra textil de vidrio-AR y para columna circular con fibra textil de carbono:

$$
\rho_{f}=\frac{4 \cdot t_{f}}{d}
$$

Cálculo del

$\alpha_{f}=\alpha_{n} \cdot \alpha_{s} \cdot \alpha_{a} \leq 1$

coeficiente

efectivo de

confinamiento

$$
\alpha_{n}=1-\frac{b^{\prime 2}+d^{\prime 2}}{3 \cdot b \cdot d}
$$

Cálculo del esfuerzo lateral en el lado " $b$ "

$$
\sigma_{l u d, b}=\alpha_{f} \cdot \rho_{f, b} \cdot f_{f d e}
$$

Cálculo de los coeficientes efectivos de confinamiento

$$
\alpha_{1 d}=2,254 \sqrt{1+7,94 \frac{\sigma_{l u d, b}}{f_{c d}}}-2 \frac{\sigma_{l u d, b}}{f_{c d}}-1,254
$$




\section{Procedimiento}

\section{Fórmula}

Cálculo del

módulo de

elasticidad del

hormigón

confinado

$$
E_{s e c, M d}=\frac{\alpha_{1 d} \cdot \alpha_{2 d} \cdot f_{c d}}{0,002 \cdot\left[1+5\left(\alpha_{1 d} \cdot \alpha_{2 d}-1\right)\right]}
$$

Cálculo del módulo de elasticidad de la camisa de TRC

$$
E_{\text {sec }, u d}=\frac{E_{c}}{1+2\left(\frac{E_{c}}{f_{c d}}-500\right) \frac{f_{f d e}}{E_{f d}}}
$$

Módulo de elasticidad de diseño del TRC, factor de reducción de capacidad para el confinamiento $\mathrm{y}_{\mathrm{E}}=1,1$

Cálculo de la deformación última del hormigón confinado

$$
\varepsilon_{c c u}=0,002\left[1+5 \cdot\left(\alpha_{1 d} \cdot \alpha_{2 d}-1\right)\right]\left[\frac{E_{s e c, M d} \cdot\left(E_{c}-E_{s e c, u d}\right)}{E_{s e c, u d} \cdot\left(E_{c}-E_{s e c, M d}\right)}\right]^{1-\frac{E_{s e c, M d}}{E_{c}}}
$$

Cálculo de la resistencia a la tracción última o de diseño

$$
\begin{gathered}
f_{c c d}=E_{s e c, u d} \cdot \varepsilon_{c c u} \geq f_{c d} \\
\varepsilon_{c c u}=\varepsilon_{c u}+k_{2}\left(\frac{\sigma_{l u d}}{f_{c d}}\right)^{n} \\
\sigma_{l u d}=\alpha_{f} \cdot \frac{(b+d)}{b \cdot d} \cdot t_{f} \cdot f_{f e}
\end{gathered}
$$

Cálculo del Espesor de TRC para Empalmes porTraslape (verificación de falla)

Cálculo del espesor tf de TRC para prevenir falla en los empalmes

$$
A_{b} \cdot f_{y} \leq p_{c} \cdot \mu \cdot \sigma_{l} \cdot l_{s}
$$$$
t_{f}=\gamma_{R d} \cdot \frac{b \cdot d \cdot\left(1-\frac{l_{s, \min }}{l_{s}}\right) \cdot A_{b} \cdot f_{y}}{\alpha_{f} \cdot(b+d) \cdot p_{c} \mu \cdot f_{f e} l_{s}}
$$

Para columna circular on fibra textil de carbono:

$$
t_{f}=\gamma_{R d} \cdot \frac{\left(\frac{\pi \cdot d^{2}}{4}\right) \cdot\left(1-\frac{l_{s, \text { min }}}{l_{s}}\right) \cdot A_{b} \cdot f_{y}}{\alpha_{f} \cdot(d \cdot \pi) \cdot p_{c} \cdot \mu \cdot f_{f e} \cdot l_{s}}
$$


y sus alcances

\begin{tabular}{|c|c|}
\hline Procedimiento & Fórmula \\
\hline $\begin{array}{l}\text { Cálculo del Espesor } \\
\text { de TRC para el } \\
\text { Pandeo de Barras de } \\
\text { Refuerzo } \\
\text { Longitudinal }\end{array}$ & $t_{f}=\frac{0,45 \cdot n \cdot f_{s}^{2} \cdot d}{4 \cdot E_{d s} \cdot E_{f} \cdot \alpha_{f}}$ \\
\hline & $E_{d s}=\frac{4 \cdot E_{s} \cdot E_{i}}{\left(\sqrt{E_{s}}+\sqrt{E_{i}}\right)^{2}}$ \\
\hline $\begin{array}{l}\text { Cálculo de carga de } \\
\text { compresión } \\
\text { soportada por el } \\
\text { hormigón }\end{array}$ & $N_{c n c}=f_{c d} \cdot A_{c u}+f_{c c d} \cdot A_{e f}$ \\
\hline $\begin{array}{l}\text { Cálculo de carga de } \\
\text { compresión axial } \\
\text { soportada por el } \\
\text { acero de refuerzo }\end{array}$ & $N_{s n}=f_{y} \cdot A_{s}$ \\
\hline $\begin{array}{l}\text { Cálculo de carga } \\
\text { última a soportar }\end{array}$ & $N_{n d}=0,85 \cdot N_{c n c}+N_{s n}$ \\
\hline $\begin{array}{l}\text { Cálculo de carga } \\
\text { última de la } \\
\text { columna "As-built }\end{array}$ & $N_{n d}=0,85 \cdot\left(A_{c c, f} \cdot f_{c d}\right)+N_{s n}$ \\
\hline
\end{tabular}

Fuente: Eurocode (2004), Caicedo (2007).

El cuadro 1 muestra la secuencia de cálculos que se realizaron para los primeros 4 escenarios, con base en la norma Eurocoge.

Cuadro 2. Procedimiento y valores considerados (según ACI)

\begin{tabular}{lc}
\hline \multicolumn{1}{c}{ Procedimiento } & \multicolumn{1}{c}{ Fórmula } \\
\hline $\begin{array}{l}\text { Cálculo de la } \\
\text { resistencia de diseño } \\
\text { existente }\end{array}$ & $\emptyset P_{n}=0,85 \cdot \emptyset \cdot\left(0,85 \cdot f^{\prime}{ }_{c}\left(A_{g}-A_{s t}\right)+f_{y} \cdot A_{s t}\right)$ \\
$\begin{array}{l}\text { Cálculo de las cargas } \\
\text { a resistir y } \\
\text { resistencia requerida }\end{array}$ & $P_{u}=1,2 \cdot(1900)+1,6 \cdot(1700)=\underline{5000 \mathrm{kN}}$ \\
\hline
\end{tabular}


Procedimiento

Verificaciones

iniciales

Cálculo de las

propiedades de diseño del sistema CFRP de confinamiento en la sección circular de la columna resistencia a compresión mayorada por el confinamiento

Cálculo de la fuerza resistente de diseño $\emptyset P_{n}=0,85 \cdot \emptyset \cdot\left(0.85 \cdot \psi \cdot f^{\prime}{ }_{c c} \cdot\left(A_{g}-A_{s t}\right)+f_{y} \cdot A_{s t}\right)$ de la estructura reforzada

Verificación de la nueva resistencia de diseño

\section{Fórmula}

$$
\emptyset P_{n}=1231,93 k N<P_{u(\text { último })}=1480 k N
$$

\section{Esfuerzo efectivo del CFRP}

$f_{f e}=C_{E} \cdot f_{f u}=0,95 \cdot 3,79 \frac{\mathrm{kN}}{\mathrm{mm}^{2}}=\underline{3,6 \mathrm{kN} / \mathrm{mm}^{2}}$.

Deformación unitaria última del CFRP

$\varepsilon_{f e}=C_{E} \cdot \varepsilon_{f u}=0,95 \cdot 0,017 \frac{\mathrm{mm}}{\mathrm{mm}}=\underline{0,016 \mathrm{~mm} / \mathrm{mm}}$

Preción de contaminación debido al CFRP

$f_{l}=\frac{f_{f e} \cdot k_{a} \cdot \rho_{f}}{2}$

Preción de contaminación debido a los atributos

$f_{l, \text { estribo }}=\frac{2 \cdot f_{f e} \cdot A_{s, \text { estr }}}{s \cdot d_{i}}$

$$
f^{\prime}{ }_{c c}=f_{c}^{\prime} \cdot\left(2,25 \cdot \sqrt{1+\frac{7,9 \cdot f^{\prime}}{f^{\prime}{ }_{c}}}-2 \cdot \frac{f^{\prime}{ }_{l}}{f^{\prime}{ }_{c}}-1,25\right)
$$

$\emptyset P_{n}=2672,26 k N$

Fuente: ACI (2005), Caicedo (2007).

El cuadro 2 muestra la secuencia de cálculos que se realizaron para los últimos 2 escenarios, con base en la norma ACI. 


\section{RESULTADOS}

Conforme la metodología planteada, se procedió a realizar cálculos matemáticos para cada uno de los 6 diseños estructurales planteados. En todos los casos se consideró que la columna a reforzar está en el estacionamiento subterráneo de un edificio de departamentos.

Los primeros dos casos muestran el diseño para refuerzo por confinamiento con textil de fibra de vidrio (para columnas de base rectangular y circular).

\section{Diseños para refuerzo por confinamiento con TRC para columna rectangular con textil de fibra de vidrio-AR, según Euro-codes}

Se presentan los datos característicos de la columna: a) longitud libre de la columna $L=3,60 \mathrm{~m} ;$ b) lado alto de la sección transversal $d=600 \mathrm{~mm}$; c) lado corto de la sección transversal $b=400 \mathrm{~mm}$; d) espacio de recubrimiento entre hormigón/acero $C=$ $30 \mathrm{~mm}$; e) redondeado de las esquinas con un radio $R=25 \mathrm{~mm}$; f) diámetro del acero de refuerzo longitudinal $\left.0_{s}=16 \mathrm{~mm} ; \mathbf{g}\right)$ diámetro del acero de refuerzo transversal $\left.0_{s, e s t r}=8 \mathrm{~mm} ; \mathbf{h}\right)$ separación entre estribos $s=$ $200 \mathrm{~mm}$; i) resistencia características del hormigón a los 28 días $f_{e k}=m_{f}\left(1-k_{n} V_{f}\right)$ [MPa]; j) resistencia a la compresión del hormigón $f=f_{c k} / 1,5$ [MPa]; k) módulo de elasticidad del hormigón $E_{e m}=9,5\left(f_{e k}+8\right)^{1 / 3}$ [GPa]; l) resistencia característica del acero de refuerzo fyk $=\operatorname{mf}(1-\mathrm{knVf})[\mathrm{MPa}]$ $y ; \mathbf{m})$ resistencia a la fluencia del acero de refuerzo fyd= fyk $/ 1,15[\mathrm{MPa}]$.

También se presentan los datos característicos del concreto de grano fino: a) cemento Portland con puzolana natural IP-30; b) tamaño de agregado máximo $\varnothing=$ $1 \mathrm{~mm}$; c) resistencia a la compresión fed,
$\mathrm{FGC}=70 \mathrm{~N} / \mathrm{mm} 2 \mathrm{y} ; \mathbf{d})$ resistencia a la tracción fyd,FGC $=5 \mathrm{~N} / \mathrm{mm} 2$. Asimismo, el textil cumplió con las siguientes características: a) diámetro de la fibra de vidrio $\varnothing=0,75 \mathrm{~mm}$; b) espesor de la capa de TRC (textil + FGC) tf $=2,75 \mathrm{~mm}$; c) resistencia a la tracción ultima ffd $=1232$ $\mathrm{N} / \mathrm{mm} 2 ;$ d) módulo de elasticidad $\mathrm{Ef}=74$ $450 \mathrm{~N} / \mathrm{mm} 2 \mathrm{y} ;$ e) deformación de rotura de $\mathrm{Ef}=0,0165$.

\section{Diseño del refuerzo por confinamiento con TRC para columna circular con fibra textil de vidrio-AR, según Euro-códigos}

En este caso, datos característicos de la columna fueron: a) longitud libre de la columna $L=2,90 \mathrm{~m}$; b) diámetro de la columna $d=300 \mathrm{~mm}$; c) diámetro inferior de la columna $d_{i}=240 \mathrm{~mm}$; d) espacio de recubrimiento entre hormigón/acero $c=25$ $\mathrm{mm}$; e) diámetro del acero de refuerzo longitudinal $?_{s}=16 \mathrm{~mm}$; f) diámetro del acero de refuerzo transversal $]_{s, e s t r}=8 \mathrm{~mm}$; g) separación entre estribos $s=150 \mathrm{~mm}$; h) características de resistencia del hormigón a los 28 días $f_{e k}=m_{f}\left(1-k_{n} V_{f}\right)[\mathrm{MPa}]$, i) resistencia a la compresión del hormigón $f=f_{c k} / 1,5$ [MPa]Módulo de elasticidad del hormigón, $E_{e m}=9,5\left(f_{e k}+\right.$ $8)^{1 / 3}[\mathrm{GPa}]$; j) resistencia característica del acero de refuerzo fyk $=\operatorname{mf}(1-\mathrm{knVf})$ [MPa] y; k) Resistencia a la fluencia del acero de refuerzo fyd=fyk1,15 [MPa].

Por su parte, los datos característicos del concreto de grano fino son: a) cemento Portland con puzolana natural IP-30; b) tamaño de agregado máximo $\emptyset=1 \mathrm{~mm}$; c) resistencia a la compresión fed, $\mathrm{FGC}=70$ $\mathrm{N} / \mathrm{mm} 2$ y; d) resistencia a la tracción fyd,FGC $=5 \mathrm{~N} / \mathrm{mm} 2$. Asimismo, los datos característicos del textil son: a) diámetro de la fibra de vidrio $\varnothing=0,75 \mathrm{~mm}$; b) 
espesor de la capa de TRC (textil + FGC) tf $=2,75 \mathrm{~mm}$; c) resistencia a la tracción ultima ffd = $1232 \mathrm{~N} / \mathrm{mm} 2$ y; d) módulo de elasticidad, $\mathrm{Ef}=74450 \mathrm{~N} / \mathrm{mm} 2$.

En la figura 1 se presenta los resultados del confinamiento de columna de sección con fibra de vidrio-AR, a la izquierda se ve la columna de sección transversal rectangular con bordes redondeados y a la derecha se presenta la columna de sección transversal circular. Se visualiza la comparación antes y después del confinamiento.
Para columna de sección transversal rectangular con bordes redondeados

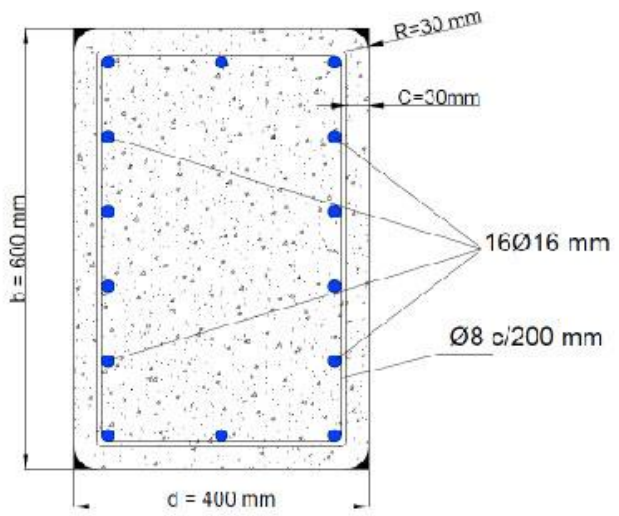

$\downarrow$

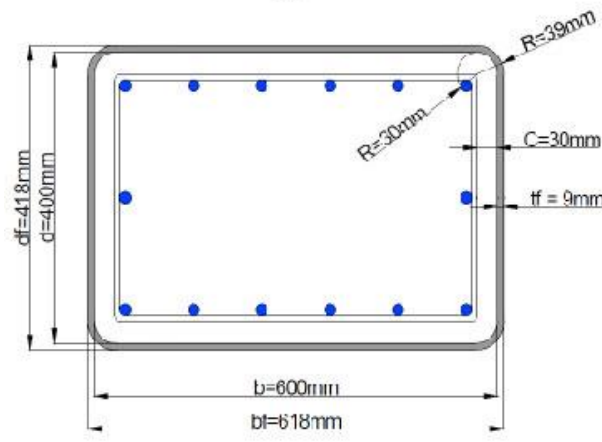

Para columna de sección transversal circularcircular
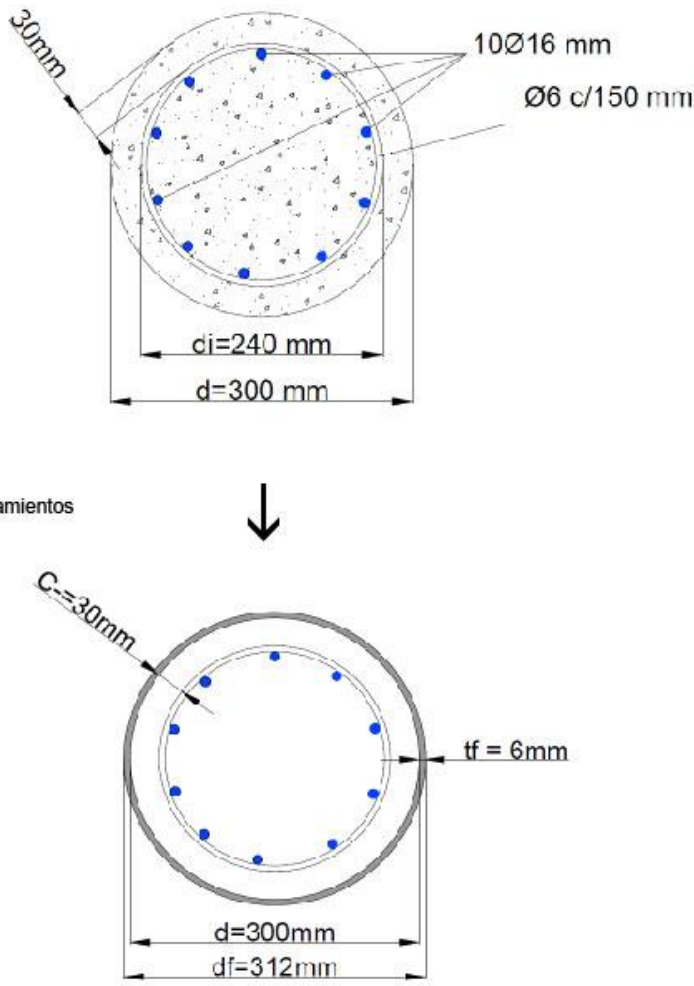

Figura 1. Resultado del confinamiento de columna de sección transversal con fibra de vidrio-AR

Como se puede ver en la figura 1, en el caso de la columna de sección rectangular, la aplicación del encamisado de TRC se traduce en una mejora de la carga axial máxima en $490,10 \mathrm{kN}$ o $7,14 \%$ comparada con la carga última de la columna antes de ser reforzada. Por su parte, en el caso de la columna de sección circular, la aplicación del encamisado de TRC se traduce en una mejora de la carga axial máxima en 1918,17 $\mathrm{kN}$ o 48,10\% comparada con la carga última de la columna antes de ser reforzada.

Continuando con el estudio, se presenta dos casos que muestran el diseño para refuerzo por confinamiento con textil de fibra de carbono (para columnas de sección rectangular y circular). 
Diseño del refuerzo por confinamiento con TRC para columna rectangular con textil de fibra de carbono, según Euro-códigos

Se presentan los datos característicos de la columna: a) longitud libre de la columna, $\mathrm{L}=3,60 \mathrm{~m}$; b) lado alto de la sección transversal, $\mathrm{d}=600 \mathrm{~mm}$; c) lado corto de la sección transversal, $\mathrm{b}=400$ $\mathrm{mm}$; d) espacio de recubrimiento entre hormigón/acero, $\mathrm{C}=30 \mathrm{~mm}$; e) redondeado de las esquinas con un radio, $\mathrm{R}$ $=25 \mathrm{~mm}$; f) diámetro del acero de refuerzo longitudinal, $\emptyset_{\mathrm{s}}=16 \mathrm{~mm}$; g) diámetro del acero de refuerzo transversal, $\emptyset_{\mathrm{s}, \mathrm{estr}}=$ $8 \mathrm{~mm} ; \mathbf{h})$ separación entre estribos, $\mathrm{s}=200$ $\mathrm{mm}$; i) característica de resistencia del hormigón a los 28 días, $\mathrm{f}_{\mathrm{ek}}=\mathrm{m}_{\mathrm{f}}\left(1-\mathrm{k}_{\mathrm{n}} \mathrm{V}_{\mathrm{f}}\right)$ [MPa]; j) resistencia a la compresión del hormigón, $\mathrm{f}=\mathrm{f}_{\mathrm{d}} / 1,5$ [MPa]; $\left.\mathbf{k}\right)$ módulo de elasticidad del hormigón, $E_{\mathrm{em}}=9,5\left(\mathrm{f}_{\mathrm{ek}}+\right.$ 8) ${ }^{1 / 3}$ [GPa]; l) resistencia característica del acero de refuerzo, fyk $=\operatorname{mf}(1-\mathrm{knVf})$ [MPa] y; $\mathbf{m})$ resistencia a la fluencia del acero de refuerzo, fyd $=f y k / 1,15$ [MPa].

Asimismo, los datos característicos del concreto de grano fino: a) cemento Portland con puzolana natural, IP-30; b) tamaño de agregado máximo, $\varnothing=1 \mathrm{~mm}$; c) resistencia a la compresión, fed,FGC = $70 \mathrm{~N} / \mathrm{mm} 2 \mathrm{y}$; d) resistencia a la tracción, fyd,FGC $=5 \mathrm{~N} / \mathrm{mm} 2$. Por su parte, los datos característicos del tipo de textil: a) diámetro de la fibra de vidrio, $\varnothing=$ $0,76 \mathrm{~mm}$; b) espesor de la capa de TRC (textil + FGC), $\mathrm{tf}=2,76 \mathrm{~mm}$; c) resistencia a la tracción ultima, $\mathrm{ffd}=1032 \mathrm{~N} / \mathrm{mm} 2$; d) módulo de elasticidad, $\mathrm{Ef}=222980$ $\mathrm{N} / \mathrm{mm} 2$ y; e) deformación de rotura, Ef $=0,0046$.

\section{Diseño del refuerzo por confinamiento con TRC para columna circular con fibra textil de carbono, según Euro-códigos}

Aquí se presentan los datos característicos de la columna: a) longitud libre de la columna, $L=2,90 \mathrm{~m}$; b) diámetro de la columna, $d=300 \mathrm{~mm}$; c) diámetro inferior de la columna, $d_{i}=240$ $\mathrm{mm}$; d) espacio de recubrimiento entre hormigón/acero, $c=25 \mathrm{~mm}$; e) diámetro del acero de refuerzo longitudinal, $?_{s}=16$ $\mathrm{mm}$; f) diámetro del acero de refuerzo transversal, $\square_{s, e s t r}=6 \mathrm{~mm} ; \mathbf{g}$ ) separación entre estribos, $s=150 \mathrm{~mm}$; h) características de resistencia del hormigón a los 28 días, $f_{e k}=m_{f}\left(1-k_{n} V_{f}\right)$ [MPa]; i) resistencia a la compresión del hormigón, $\left.f=f_{c k} / 1,5[\mathrm{MPa}] ; \mathbf{j}\right)$ módulo de elasticidad del hormigón, $E_{e m}=9,5\left(f_{e k}+\right.$ ${ }_{8)}^{1 / 3}$ [GPa]; k) resistencia característica del acero de refuerzo, fyk $=\operatorname{mf}(1-\mathrm{knVf})$ [MPa] y; l) resistencia a la fluencia del acero de refuerzo, fyd $=$ fyk/1,15 [MPa].

De la misma manera se presentan los datos característicos del concreto de grano fino: a) cemento Portland con puzolana natural, IP-30; b) tamaño de agregado máximo, $\varnothing=1 \mathrm{~mm} ;$ c) resistencia a la compresión, fed, FGC = $70 \mathrm{~N} / \mathrm{mm} 2 \mathrm{y}$; d) resistencia a la tracción, fyd,FGC $=5 \mathrm{~N} / \mathrm{mm} 2$. Por su parte, los datos característicos del tipo de textil: a) diámetro de la fibra de vidrio, $\varnothing=$ $0,75 \mathrm{~mm}$; b) espesor de la capa de TRC (textil + FGC), $\mathrm{tf}=2,76 \mathrm{~mm}$; c) resistencia a la tracción ultima, ffd $=1032 \mathrm{~N} / \mathrm{mm} 2$ y; d) módulo de elasticidad, $\mathrm{Ef}=222980$ $\mathrm{N} / \mathrm{mm} 2$. 
En la figura 2 se presenta los resultados del confinamiento de columna de sección transversal con fibra de carbono, a la izquierda se ve la columna de sección transversal rectangular con bordes redondeados y a la derecha se presenta la columna de sección

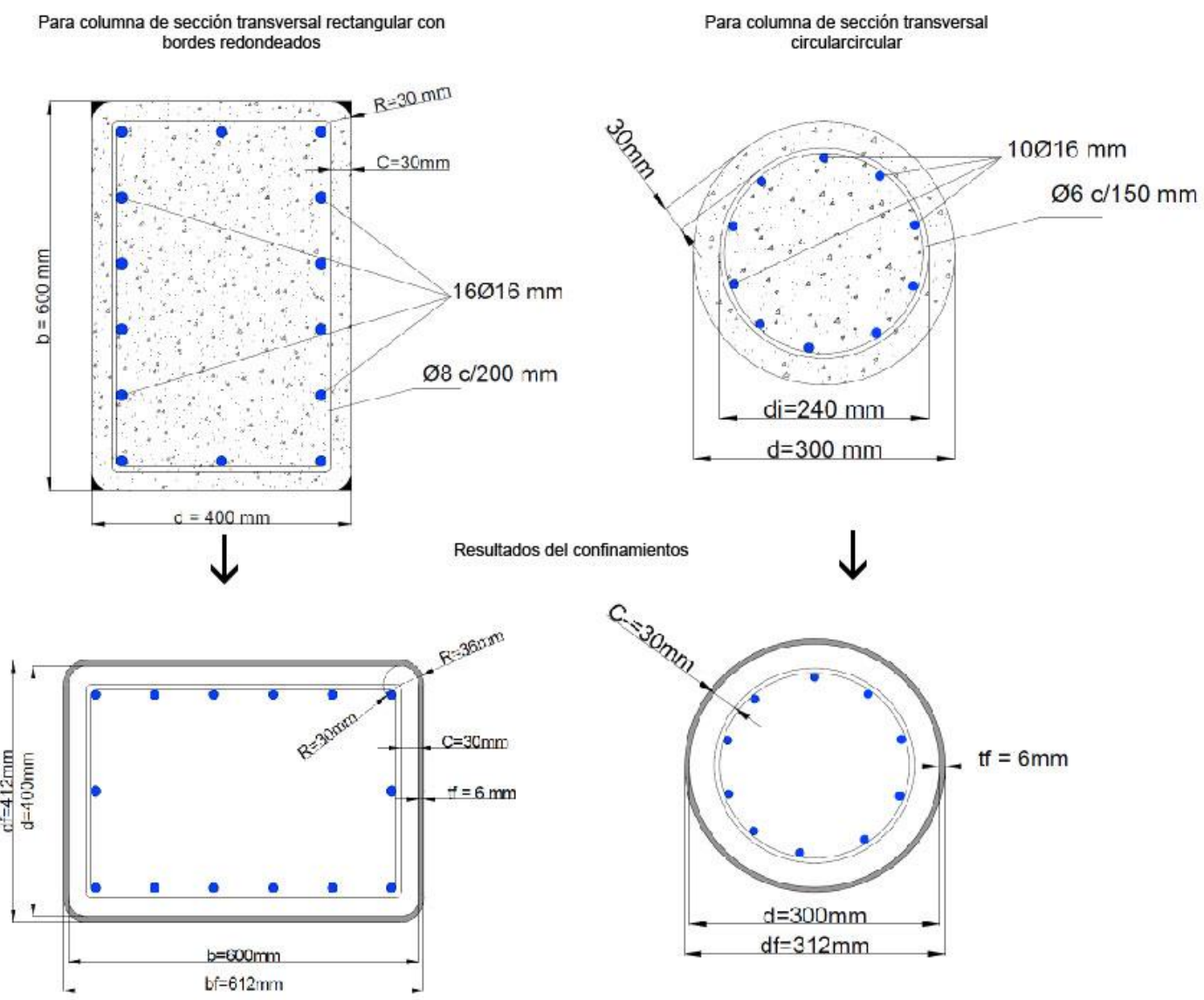

transversal circular. Se visualiza la comparación antes y después del confinamiento.

Figura 2. Resultado del Confinamiento de columna de sección transversal con fibra de carbono

La figura 2 permite comprender que en el caso de la columna de sección rectangular la aplicación del encamisado de TRC se traduce en una mejora de la carga axial máxima en $392,09 \mathrm{kN}$ o 5,80 \% comparada con la carga última de la columna antes de ser reforzada. Por su parte, en la parte derecha de la figura, se aprecia que, en el caso de la columna circular, la aplicación del encamisado de TRC se traduce en una mejora de la carga axial máxima en 1904,74 kN o 47,92 \% comparada con la carga última de la columna antes de ser reforzada. 
Diseño del Refuerzo con CFRP por Confinamiento para una Columna Rectangular, según norma ACI

Los datos característicos de la columna son: a) longitud libre de la columna, $L=3,60 \mathrm{~m}$; b) dimensión de la columna, $h=600 \mathrm{~mm}$; c) dimensión de la columna, $b=400 \mathrm{~mm}$; d) área de acero de refuerzo longitudinal, $A_{s t}=33,16 \mathrm{~cm}^{2}$; e) área de acero de refuerzo a cortante, $\mathrm{A}_{s, \text { sestr }}$ $=1,00 \mathrm{~cm}^{2} ; \mathbf{f}$ ) separación entre estribos, $s=$ $200 \mathrm{~mm}$; g) resistencia a la compresión del hormigón, f re $=25 \mathrm{~N} / \mathrm{mm} 2$; h) módulo de elasticidad del hormigón, Ee $=4740$

$\mathrm{f}^{\prime} \mathrm{e}[\mathrm{N} / \mathrm{mm} 2]$; i) módulo de elasticidad del hormigón, Ee $=4740 \cdot \checkmark \mathrm{f}^{\prime} \mathrm{e}[\mathrm{N} / \mathrm{mm} 2]$; j) módulo de elasticidad del hormigón, $E_{e m}=$ $\left.9,5\left(f_{e k}+8\right)^{1 / 3} \quad[\mathrm{GPa}] ; \mathbf{k}\right)$ resistencia característica del acero de refuerzo, fyk = $\operatorname{mf}(1$ - knVf) [MPa] y; l) resistencia a la fluencia del acero de refuerzo, fyd= fyk/1,15 [MPa]. Por su parte, los datos característicos de la manta de fibra de carbono son: a) espesor de la capa, $\mathrm{tf}=$ $0,165 \mathrm{~mm}$; b) resistencia a la tracción ultima, $f_{f u}=3790 \mathrm{~N} / \mathrm{mm} 2 ; \quad$ c) deformación de rotura, $\mathrm{Efu}=0,017$ $\mathrm{mm} / \mathrm{mm}$ y; d) módulo de elasticidad, $E_{f}=$ $228 \mathrm{kNmm}^{2}$.

\section{Diseño del Refuerzo con CFRP por Confinamiento para una Columna Circular, según norma ACI}

Finalmente, los datos característicos de la columna fueron: a) longitud libre de la columna, $H=2,90 \mathrm{~m}$; b) dimensión de la columna, $h=300 \mathrm{~mm}$; c) dimensión de la columna, $b=240 \mathrm{~mm}$; d) área de acero de refuerzo longitudinal, $A_{s t}=21,11 \mathrm{~cm}^{2}$; e) área de acero de refuerzo a cortante, $\mathrm{A}_{s, \text { estr }}$ $=0,56 \mathrm{~cm}^{2} ; \mathbf{f}$ ) separación entre estribos, $s=$ $150 \mathrm{~mm}$; g) resistencia a la compresión del hormigón, f re $=21 \mathrm{~N} / \mathrm{mm} 2$; h) módulo de elasticidad del hormigón, Ee $=4740$ $\mathrm{f}^{\prime} \mathrm{e}[\mathrm{N} / \mathrm{mm} 2]$; i) módulo de elasticidad del hormigón, Ee $=420 \checkmark \mathrm{f}^{\prime} \mathrm{e}[\mathrm{N} / \mathrm{mm} 2]$. En el caso de la manta de fibra de carbono, los datos característicos fueron: a) espesor de la capa, $\mathrm{tf}=0,165 \mathrm{~mm}$; b) resistencia a la tracción ultima, $f_{f u}=3790 \mathrm{~N} / \mathrm{mm} 2$; c) deformación de rotura, $\mathrm{Efu}=0,017$ $\mathrm{mm} / \mathrm{mm}$ y; d) módulo de elasticidad, $E_{f}=$ $228 \mathrm{kNmm}^{2}$.

En la figura 3 se presenta los resultados del Refuerzo con CFRP por Confinamiento, a la izquierda se ve la columna de sección rectangular y a la derecha se presenta la columna de sección circular. Se visualiza la comparación antes y después del confinamiento. 


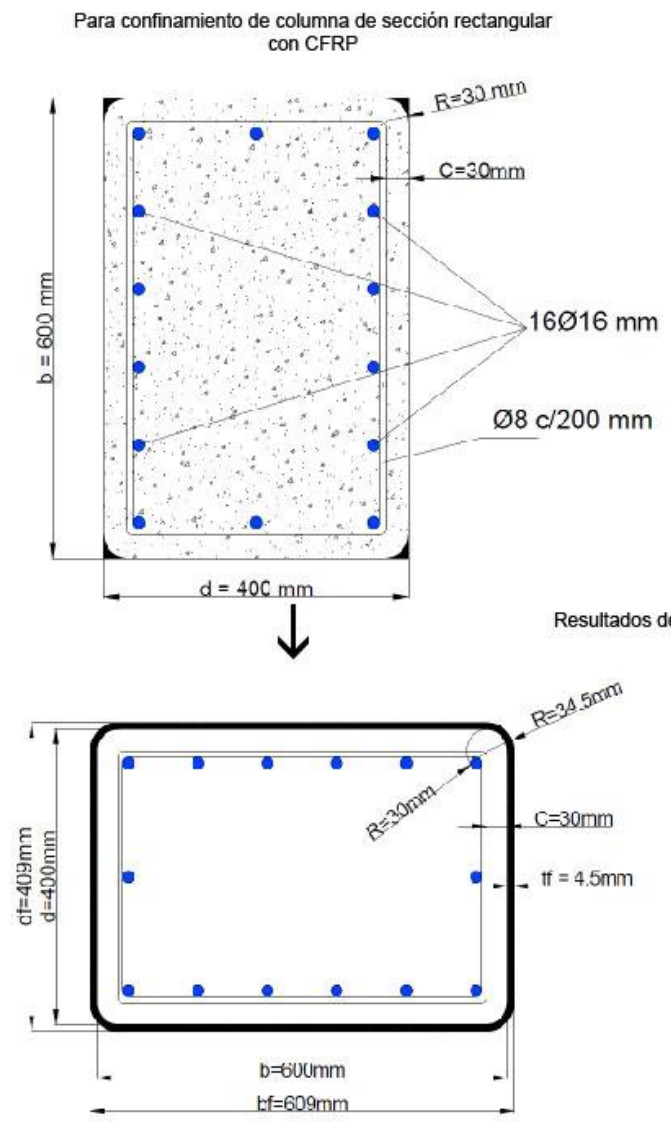

Figura 3. Refuerzo con CFRP por Confinamiento

Al lado izquierdo de la figura 3 se parecía que en la columna de sección rectangular la estructura reforzada con CFRP soporta el nuevo incremento de carga axial con un incremento de $1.69 \%$ en la resistencia. Por su parte, en el caso de la columna circular, la estructura reforzada con CFRP soporta el nuevo incremento de carga axial. Usando una sola capa del sistema MBrase $\mathrm{C}-130$ es posible resistir el incremento de carga axial en las columnas circulares con un incremento del $44,7 \%$ en la resistencia (figura 3).
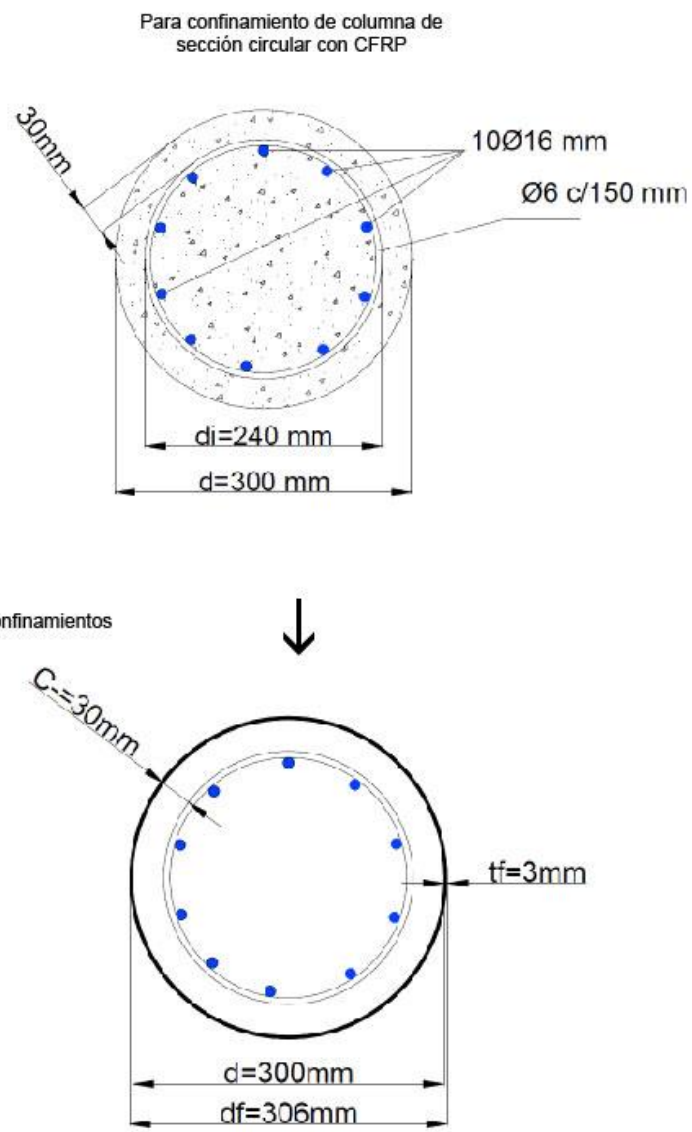

Un análisis comparativo permitió contrastar las principales ventajas $\mathrm{y}$ desventajas de cada diseño, considerando variables de eficiencia tales como resistencia a la tracción última, módulo de elasticidad, deformación de rotura, número de capas de refuerzo, espesor de la capa final, incremento de carga en [kN], incremento de carga en [\%] y precio por el refuerzo. Según se muestra en el cuadro 3. 
Cuadro 3. Sístesis y comparación de los resultados obtenidos

\begin{tabular}{|c|c|c|c|}
\hline CARACTERÍSTICAS & $\begin{array}{l}\text { COLUMNA } \\
\text { CIRCULAR }\end{array}$ & $\begin{array}{l}\text { COLUMNA } \\
\text { CIRCULAR }\end{array}$ & $\begin{array}{l}\text { COLUMNA } \\
\text { CIRCULAR }\end{array}$ \\
\hline Longitud libre de columna & $\mathrm{L}=2,90 \mathrm{~m}$ & $\mathrm{~L}=2,90 \mathrm{~m}$ & $\mathrm{~L}=2,90 \mathrm{~m}$ \\
\hline Dimensiones de la columna & $\mathrm{D}=300 \mathrm{~mm}$ & $\mathrm{D}=300 \mathrm{~mm}$ & $\mathrm{D}=300 \mathrm{~mm}$ \\
\hline Espacio de recubrimiento & $\mathrm{C}=30 \mathrm{~mm}$ & $\mathrm{C}=30 \mathrm{~mm}$ & $\mathrm{C}=30 \mathrm{~mm}$ \\
\hline $\begin{array}{l}\text { Radio de redondeo de } \\
\text { esquinas }\end{array}$ & - & - & - \\
\hline $\begin{array}{l}\text { Diámetro del acero de } \\
\text { refuerzo longitudinal }\end{array}$ & $\emptyset=10 \mathrm{~mm}$ & $\emptyset=10 \mathrm{~mm}$ & $\emptyset=10 \mathrm{~mm}$ \\
\hline $\begin{array}{l}\text { Diámetro del acero de } \\
\text { refuerzo transversal }\end{array}$ & $\emptyset=6 \mathrm{~mm}$ & $\emptyset=6 \mathrm{~mm}$ & $\emptyset=6 \mathrm{~mm}$ \\
\hline Separación entre estribos & $\mathrm{S}=150 \mathrm{~mm}$ & $\mathrm{~S}=150 \mathrm{~mm}$ & $\mathrm{~S}=150 \mathrm{~mm}$ \\
\hline $\begin{array}{l}\text { Resistencia a la compresión } \\
\text { del hormigón }\end{array}$ & $\mathrm{fcd}=21 \mathrm{MPa}$ & $\mathrm{fcd}=21 \mathrm{MPa}$ & $\mathrm{f}^{\prime} \mathrm{c}=21 \mathrm{MPa}$ \\
\hline $\begin{array}{l}\text { Módulo de elasticidad del } \\
\text { hormigón }\end{array}$ & $\mathrm{Ecm}=32220$ & $\mathrm{Ecm}=32220$ & $\mathrm{Ec}=32220$ \\
\hline $\begin{array}{l}\text { Resistencia a la tracción del } \\
\text { acero }\end{array}$ & $\mathrm{fy}=400 \mathrm{MPa}$ & $\mathrm{fy}=400 \mathrm{MPa}$ & $\mathrm{fy}=400 \mathrm{MPa}$ \\
\hline $\begin{array}{l}\text { Resistencia a la fluencia del } \\
\text { acero }\end{array}$ & $\mathrm{fu}=250 \mathrm{MPa}$ & $\mathrm{fu}=250 \mathrm{MPa}$ & $\mathrm{fu}=250 \mathrm{MPa}$ \\
\hline Tipo de aglomerante & $\begin{array}{l}\text { Concreto de grano } \\
\text { fino }\end{array}$ & $\begin{array}{l}\text { Concreto de grano } \\
\text { fino }\end{array}$ & Pegamento epoxico \\
\hline Tipo de refuerzo & $\begin{array}{l}\text { Textil de fibra de } \\
\text { vidrio-AR }\end{array}$ & $\begin{array}{l}\text { Textil de fibra de } \\
\text { carbono }\end{array}$ & Manta de carbono \\
\hline Diámetro de la fibra & $\emptyset=0,75 \mathrm{~mm}$ & $\emptyset=0,76 \mathrm{~mm}$ & $\emptyset=0,165 \mathrm{~mm}$ \\
\hline Espesor de la capa & $\mathrm{tf}=2,75 \mathrm{~mm}$ & $\mathrm{tf}=2,76 \mathrm{~mm}$ & $\mathrm{tf}=1,2 \mathrm{~mm}$ \\
\hline $\begin{array}{l}\text { Resistencia a la tracción } \\
\text { última }\end{array}$ & $\mathrm{ffd}=1232 \mathrm{MPa}$ & $\mathrm{ffd}=1032 \mathrm{MPa}$ & $\mathrm{ffu}=3790 \mathrm{MPa}$ \\
\hline Módulo de elasticidad & $\mathrm{Ef}=74450 \mathrm{MPa}$ & $\mathrm{Ef}=222980 \mathrm{MPa}$ & $\mathrm{Ef}=222941 \mathrm{MPa}$ \\
\hline Deformación de rotura & $\varepsilon f=0,0165$ & $\varepsilon f=0,0046$ & $\varepsilon f=0,017$ \\
\hline Número de capas de refuerzo & 2 & 2 & 2 \\
\hline Espesor de la capa final & $\mathrm{t}^{\prime} \mathrm{s}=6 \mathrm{~mm}$ & $\mathrm{t}^{\prime} \mathrm{s}=6 \mathrm{~mm}$ & $\mathrm{t}^{\prime} \mathrm{s}=3 \mathrm{~mm}$ \\
\hline Incremento de carga en $[\mathrm{kN}]$ & $1918,17 \mathrm{kN}$ & $1904.74 \mathrm{kN}$ & $1382.32 \mathrm{kN}$ \\
\hline Incremento de carga en [\%] & $48,1 \%$ & $47,92 \%$ & $48,3 \%$ \\
\hline
\end{tabular}




\begin{tabular}{|c|c|c|c|}
\hline CARACTERÍSTICAS & $\begin{array}{l}\text { COLUMNA } \\
\text { CIRCULAR }\end{array}$ & $\begin{array}{l}\text { COLUMNA } \\
\text { CIRCULAR }\end{array}$ & $\begin{array}{l}\text { COLUMNA } \\
\text { CIRCULAR }\end{array}$ \\
\hline $\begin{array}{l}\text { PRECIO POR EL REFUERZO } \\
\text { (bolivianos) }\end{array}$ & 430,49 & 1119,10 & 7995,45 \\
\hline Longitud libre de columna & $\mathrm{L}=3,60 \mathrm{~m}$ & $\mathrm{~L}=3,60 \mathrm{~m}$ & $\mathrm{~L}=3,60 \mathrm{~m}$ \\
\hline Dimensiones de la columna & $\begin{array}{c}\mathrm{b}=400 \mathrm{~mm} ; \mathrm{d}=600 \\
\mathrm{~mm}\end{array}$ & $\begin{array}{c}\mathrm{b}=400 \mathrm{~mm} ; \mathrm{d}=600 \\
\mathrm{~mm}\end{array}$ & $\begin{array}{c}\mathrm{b}=400 \mathrm{~mm} ; \mathrm{d}=600 \\
\mathrm{~mm}\end{array}$ \\
\hline Espacio de recubrimiento & $\mathrm{C}=30 \mathrm{~mm}$ & $\mathrm{C}=30 \mathrm{~mm}$ & $\mathrm{C}=30 \mathrm{~mm}$ \\
\hline $\begin{array}{l}\text { Radio de redondeo de } \\
\text { esquinas }\end{array}$ & $\mathrm{R}=30 \mathrm{~mm}$ & $\mathrm{R}=30 \mathrm{~mm}$ & $\mathrm{R}=30 \mathrm{~mm}$ \\
\hline $\begin{array}{l}\text { Diámetro del acero de } \\
\text { refuerzo longitudinal }\end{array}$ & $\emptyset=16 \mathrm{~mm}$ & $\varnothing=16 \mathrm{~mm}$ & $\emptyset=16 \mathrm{~mm}$ \\
\hline $\begin{array}{l}\text { Diámetro del acero de } \\
\text { refuerzo transversal }\end{array}$ & $\emptyset=8 \mathrm{~mm}$ & $\emptyset=8 \mathrm{~mm}$ & $\emptyset=8 \mathrm{~mm}$ \\
\hline Separación entre estribos & $\mathrm{S}=200 \mathrm{~mm}$ & $\mathrm{~S}=200 \mathrm{~mm}$ & $\mathrm{~S}=200 \mathrm{~mm}$ \\
\hline $\begin{array}{l}\text { Resistencia a la compresión } \\
\text { del hormigón }\end{array}$ & $\mathrm{fcd}=25 \mathrm{MPa}$ & $\mathrm{fcd}=25 \mathrm{MPa}$ & $\mathrm{f}^{\prime} \mathrm{c}=25 \mathrm{MPa}$ \\
\hline $\begin{array}{l}\text { Módulo de elasticidad del } \\
\text { hormigón }\end{array}$ & $\mathrm{Ecm}=34000 \mathrm{MPa}$ & $\mathrm{Ecm}=34000 \mathrm{MPa}$ & $\mathrm{Ec}=34000 \mathrm{MPa}$ \\
\hline $\begin{array}{l}\text { Resistencia a la tracción del } \\
\text { acero }\end{array}$ & $\mathrm{fy}=400 \mathrm{MPa}$ & $\mathrm{fy}=400 \mathrm{MPa}$ & $\mathrm{fy}=400 \mathrm{MPa}$ \\
\hline $\begin{array}{l}\text { Resistencia a la fluencia del } \\
\text { acero }\end{array}$ & $\mathrm{fu}=250 \mathrm{MPa}$ & $\mathrm{fu}=250 \mathrm{MPa}$ & $\mathrm{fu}=250 \mathrm{MPa}$ \\
\hline Tipo de aglomerante & $\begin{array}{l}\text { Concreto de grano } \\
\text { fino }\end{array}$ & $\begin{array}{l}\text { Concreto de grano } \\
\text { fino }\end{array}$ & Pegamento epóxico \\
\hline Tipo de refuerzo & $\begin{array}{l}\text { Textil de fibra de } \\
\text { vidrio-AR }\end{array}$ & $\begin{array}{l}\text { Textil de fibra de } \\
\text { carbono }\end{array}$ & Manta de carbono \\
\hline Diámetro de la fibra & $\varnothing=0,75 \mathrm{~mm}$ & $\varnothing=0,76 \mathrm{~mm}$ & $\varnothing=0,165 \mathrm{~mm}$ \\
\hline Espesor de la capa & $\mathrm{tf}=2,75 \mathrm{~mm}$ & $\mathrm{tf}=2,76 \mathrm{~mm}$ & $\mathrm{tf}=1,2 \mathrm{~mm}$ \\
\hline $\begin{array}{l}\text { Resistencia a la tracción } \\
\text { última }\end{array}$ & $\mathrm{ffd}=1232 \mathrm{MPa}$ & $\mathrm{ffd}=1032 \mathrm{MPa}$ & $\mathrm{ffu}=3790 \mathrm{MPa}$ \\
\hline Módulo de elasticidad & $\mathrm{Ef}=74450 \mathrm{MPa}$ & $\mathrm{Ef}=222980 \mathrm{MPa}$ & $\mathrm{Ef}=222941 \mathrm{MPa}$ \\
\hline Deformación de rotura & $\varepsilon f=0,0165$ & $\varepsilon f=0,0046$ & $\varepsilon f=0,017$ \\
\hline Número de capas de refuerzo & 3 & $\begin{array}{c}\text { adoptado } 2 \text { con } 1 \\
\text { cumple }\end{array}$ & 3 \\
\hline Espesor de la capa final & $\mathrm{t}^{\prime} \mathrm{s}=9 \mathrm{~mm}$ & $\mathrm{t}^{\prime} \mathrm{s}=6 \mathrm{~mm}$ & $\mathrm{t}^{\prime} \mathrm{s}=4.5 \mathrm{~mm}$ \\
\hline $\begin{array}{l}\text { Precio por el refuerzo } \\
\text { (bolivianos) }\end{array}$ & 1664,78 & 4388,98 & 6800,00 \\
\hline
\end{tabular}


En el cuadro 3 se advierte que la mejor alternativa para reforzar columnas de hormigón armado es el concreto de grano fino combinado con fibra textil (de vidrio o carbono). Esta composición de materiales: 1) incrementa su capacidad de carga, 2) reduce la probabilidad de falla por cortante, 3) reduce la probabilidad de falla deformación, 4) incrementa la resistencia a falla de puntos de empalme por traslape, 5) incrementa la resistencia a falla por pandeo $y, 6)$ reduce el precio.

\section{CONCLUSIONES}

Este estudio esta direccionado a su aplicación en Bolivia. En primer lugar, porque se estudió la forma de aplicar y elaborar el concreto de grano fino con cementos del medio, encontrando las cantidades de dosificación, con aditivos puzolánicos y plastificantes, necesarios para la estabilización de la matriz de concreto. En segundo lugar, porque se dan a conocer los diferentes tipos de textil, sus características y su forma de aplicación en el refuerzo. Añadiendo que es posible la importación de las fibras textiles de vidrio AR por su bajo costo, ya que el actual método de refuerzo con CFRP epóxico importa todos los aditamentos por medio de SIKA Bolivia S.A. para el refuerzo de columnas por confinamiento y son de costo elevado. Finalmente, los cálculos desarrollados sirven como una guía de cálculo para la predicción del comportamiento del refuerzo con TRC, además de las comparaciones en las filosofías de cálculo utilizadas por el EuroCódigo y ACI que hacen posible el entendimiento y desarrollo de este.

El refuerzo y la rehabilitación de columnas estructurales de hormigón armado mediante el encamisado con Fibra
Textil, tanto de vidrio como de carbono, en combinación con Concreto de Grano Fino (FGC), no solo proporcionan confinamiento al envolverse sobre la superficie de la columna, incrementado su capacidad de carga y capacidad de deformación, sino que también incrementa el confinamiento en los puntos de empalme por traslape y la probabilidad de falla en los mismos. De la misma forma, se ha investigado que se incrementa la resistencia a la falla por pandeo del acero de refuerzo longitudinal y también la resistencia a las fuerzas cortantes y a su probabilidad de falla por cortante.

El refuerzo de columnas de sección circular es mucho más efectivo, por ambos métodos, debido a que su forma geométrica permite un total confinamiento del área efectiva, además de que las fuerzas laterales proporcionadas por el encamisado actúan de manera uniforme y continua alrededor del perímetro de la circunferencia. En cambio, en columnas de sección transversal rectangular y/o cuadrada, el confinamiento se ve afectado en la zona de las esquinas, ya que el encamisado solo actúa aportando resistencia y esfuerzos laterales en las puntas de las esquinas, es por esta razón es que el redondeado de las esquinas en este tipo de secciones es necesario, ya que aumentan la superficie de contacto del encamisado y por lo tanto los esfuerzos laterales y área de confinamiento se ven incrementadas.

Otra razón para que el refuerzo con encamisado de TRC para columnas de sección circular alcance un mayor incremento en la resistencia a la compresión que el encamisado con CFRP, se debe a que la combinación de sus materiales aprovecha y combina mejor las 
propiedades de estos, permitiendo que cada uno aporte con sus características de resistencia y su comportamiento sea elástico hasta la rotura.

En cuanto a lo que se refiere a los diferentes tipos de textil que se pueden utilizar en el refuerzo con TRC, la fibra de carbono tiene una mayor resistencia a la tracción que la fibra de vidrio-AR. Sin embargo, la deformación última de la fibra de vidrio- AR es mayor que la fibra de carbono y su adherencia con aglomerante inorgánico es mucho mayor debido a grado de succión capilar que presenta la fibra de vidrio, sin dejar de mencionar su bajo costo incluso en comparación con el acero.

Para que el efecto del confinamiento sea eficaz cuando se utiliza CFRP, es necesario que la sección transversal sea circular (preferentemente) y si es poligonal su relación de lados no deberá ser mucho mayor la unidad $(1,0 \leq \mathrm{b} / \mathrm{d} \leq 1,10)$. Entre las principales ventajas del uso de bandas o láminas de fibras reforzadas con polímeros se encuentran: inmunidad a la corrosión, bajo peso (alrededor de $1 / 4$ de el del acero), facilidad de aplicación en espacios reducidos, no se requiere vibrado para su aplicación, reducción de los tiempos de mano de obra, elevada resistencia a la tracción, facilidad de adaptación a diversas formas geométricas.

Algunos de los inconvenientes que puede tener esta técnica de refuerzo: baja resistencia al fuego, baja resistencia al vandalismo o las acciones accidentales, baja resistencia en el caso que se deje el material sin protección superficial.

Si se expone a los CFRP a elevadas temperaturas, como por ejemplo en un incendio, se puede llegar a una degradación prematura que desencadena en el colapso (algunas resinas epóxicas comienzan a ablandarse entre los 45 y $70^{\circ} \mathrm{C}$ ). De ahí que los materiales poliméricos reforzados con fibra no siempre puedan ser aplicados en sustitución de otros sistemas de refuerzo. El costo de los materiales por unidad de peso es mucho mayor si se lo compara con el acero y el textil de fibra de vidrio-AR (no así en términos de resistencia). Y por último este material compuesto presenta la desventaja de tener coeficientes de dilatación térmica diferentes a los del hormigón.

Los problemas comúnmente asociados con resinas epóxicas (CFRP), tales como el mal comportamiento a altas temperaturas, altos costos iniciales, los riesgos tóxicos para los trabajadores, la incompatibilidad con ciertos sustratos, dificultad en la aplicación en tiempo frío e inaplicabilidad en superficies mojadas, han llevado a la idea de la sustitución de la resina epóxica toxica y la fibra de carbono con alto costo, por un textil de fibra de vidrio-AR de bajo costo y un concreto de grano fino como ligante, más amigables con el medio ambiente, resistentes y durables en el tiempo, sin problemas de corrosión y mantenimiento casi nulo.

El método de encamisado por confinamiento se aplica de manera óptima en secciones circulares. Cuando se trata del confinamiento de una sección cuadrada o rectangular, el refuerzo comienza a perder eficacia y el área efectivamente confinada se reduce a una porción menor del área encerrada por los cercos. Para el armado de barras longitudinales, la zona confinada queda delimitada por parábolas de descarga que se "apoyan" en esta armadura. Para los refuerzos con TRC, se redondean a modo de ochavas en las esquinas de las secciones rectangulares para que las 
parábolas de descarga nazcan de estas zonas con mayor longitud de contacto. El radio mínimo de redondeo es de $20 \mathrm{~mm}$, tanto para columnas cuadradas como rectangulares.

La aplicación de las camisas de TRC por el método de laminación para el refuerzo de las columnas por confinamiento, requiere de una preparación previa de la superficie de la columna antes de ser aplicada la primera capa de refuerzo. Es necesario un correcto lijado de la superficie del hormigón viejo para garantizar una buena unión y evitar algún tipo de falla por desprendimiento o desunión.

La distancia de traslape o longitud de solapamiento mínima para el aplicado de capas textiles por el método de laminación es de 150-200 mm.

Cuando se utilizan textiles bi-axiales con envoltura total de la columna reforzada, las fibras de urdimbre deben ser colocadas en dirección perpendicular al eje axial de la columna.

Un buen control en la dosificación de la elaboración del concreto de grano fino será exigido, ya que será requerida una alta consistencia de fluidez en la matriz para poder obtener completa penetración en el textil y a manera de garantizar una buena unión. Así como también, un buen comportamiento de carga, buenas propiedades mecánicas del compuesto y la durabilidad del material de refuerzo.

\section{REFERENCIAS}

ACI. (2005). Requisitos de reglamento para concreto estructural y comentario. In A. 318S-05 México city, México: ACI Committee 318, Instituto Mexicano del Cemento y del Concreto

Alzate, A. (2012). Análisis de los modelos de comportamiento de vigas de hormigón armado reforzadas a cortante con polímeros armados con fibras (FRP). Madrid: Universidad Politécnica de Madrid

Brameshuber, W. (2006). Report 36: textile reinforced concrete-state-of-the-art report of RILEM TC 201-TRC (Vol. 36). RILEM publications

Butrón, B. (2011). Aplicación de polímeros reforzados con fibra de carbono para el refuerzo hormigón armado. Cochabamba, Bolivia: Universidad Católica San Pablo.

Caicedo, N., y Triantafillou, T. C. (2007). Use of frp and trm jackets for ductility in. Patras: University of Patras

Cubillos, P., y Montoya J. (2019). Efectos de las técnicas $\mathrm{y}$ materiales sobre el reforzamiento de la mampostería, y su incidencia en su comportamiento

Hegger, J., y Voss, S. (2008). Investigations on the bearing behaviour and application potential of textile reinforced concrete. Engineering structures

Schanack, F., y Muñoz, C. (2006). Estructuras de hormigón con armadura textil 